\title{
INDEFINITE ELLIPTIC BOUNDARY VALUE PROBLEMS ON IRREGULAR DOMAINS
}

\author{
JACQUELINE FLECKINGER AND MICHEL L. LAPIDUS
}

(Communicated by Palle E. T. Jorgensen)

\begin{abstract}
We establish estimates for the remainder term of the asymptotics of the Dirichlet or Neumann eigenvalue problem

$$
-\Delta u(x)=\lambda r(x) u(x), \quad x \in \Omega \subset \mathbb{R}^{n},
$$

defined on the bounded open set $\Omega \subset \mathbb{R}^{n}$; here, the "weight" $r$ is a real-valued function on $\Omega$ which is allowed to change sign in $\Omega$ and the boundary $\partial \Omega$ is irregular. We even obtain error estimates when the boundary is "fractal".

These results-which extend earlier work of the authors [particularly, J. Fleckinger \& M. L. Lapidus, Arch. Rational Mech. Anal. 98 (1987), 329356; M. L. Lapidus, Trans. Amer. Math. Soc. 325 (1991), 465-529]-are already of interest in the special case of positive weights.
\end{abstract}

\section{INTRODUCTION}

In this paper, we study the influence of the irregularity of the weight $r$ and of the boundary $\partial \Omega$ on the asymptotics of the eigenvalues for the following boundary value problem (in its variational formulation):

$$
-\Delta u=\lambda r u \quad \text { in } \Omega \subset \mathbb{R}^{n},
$$

with Dirichlet or Neumann homogeneous boundary conditions.

Here, $\Omega$ is a bounded open set and $r$ is a real-valued function on $\Omega$ that is allowed to change sign in $\Omega$ (in which case it is called an "indefinite weight function") and may be discontinuous. Under suitable assumptions on $r$ and $\Omega$ ([P1], [BS], [FF], [L1], [FL1], ... ), there exists a countable sequence of positive [resp., negative] eigenvalues tending to $+\infty$ [resp., $-\infty$ ]; furthermore,

Received by the editors May 19, 1993.

1991 Mathematics Subject Classification. Primary 35J05, 35J20, 35P20; Secondary 28A75, 31B20, 31B35, 41A46, 47A70.

Key words and phrases. Dirichlet and Neumann Laplacians, indefinite elliptic problems, variational boundary value problems on irregular domains, indefinite weight functions, asymptotics of eigenvalues, remainder estimate, interplay between the irregularity of the weight and of the boundary, Whitney-type coverings, "fractal" boundaries, vibrations of drums with fractal boundary and with variable mass density.

The second author's research was partially supported by the National Science Foundation under Grants DMS-8904389 (transferred to the University of California as NSF Grant DMS-9196085) and DMS-9207098, as well as by the M. G. Michael Award for Excellence in Research from the Franklin College of Arts and Sciences of the University of Georgia, and by Yale University. 
the number of positive eigenvalues less than $\lambda$ for the above problem, denoted by $N_{i}^{+}(\lambda ; r, \Omega)$ (where $i=0$ [resp., $i=1$ ] corresponds to the Dirichlet [resp., Neumann] problem) satisfies

$$
\text { (1.1) } N_{i}^{+}(\lambda ; r, \Omega) \sim W\left(\lambda ; r_{+}, \Omega\right):=(2 \pi)^{-n} \mathscr{B}_{n} \lambda^{n / 2} \int_{\Omega} r_{+}^{n / 2}, \text { as } \lambda \rightarrow+\infty \text {, }
$$

where $r_{+}=\max (r, 0)$ and $\mathscr{B}_{n}$ is the volume of the unit ball in $\mathbb{R}^{n}$.

Of course, we also introduce $N_{i}^{-}(\lambda ; r, \Omega)$, the number of negative eigenvalues larger than $\lambda<0$.

We study here the asymptotics of the "remainder term":

$$
R_{i}^{ \pm}(\lambda ; r, \Omega):=N_{i}^{ \pm}(\lambda ; r, \Omega)-W\left(\lambda ; r_{ \pm}, \Omega\right)
$$

and extend (in the special case of the Dirichlet and Neumann Laplacians) earlier results of the authors. In particular, we obtain estimates for the "remainder term" of $(\mathbf{P})$ valid when $\partial \Omega$ is very irregular and even "fractal". We thereby obtain-as was suggested in [L2, Remark 3.5(c), p. 490]-the counterpart of [FL2, Theorem 2, p. 339] and [L2, Theorems 2.3 and 4.1, pp. 482-483 and pp. 510-511] for the Dirichlet and Neumann Laplacians with an "indefinite weight function" and on an open set with "rough boundary". In the former case [FL2], the weight function $r$ is allowed to be indefinite and discontinuous but the boundary $\partial \Omega$ is assumed to be (relatively) regular, whereas in the latter case [L2], the boundary is allowed to be "fractal" but $r \equiv 1$.

Our results show, in particular, that there is an interesting interplay between the singularities (or the oscillations) of the weight function $r$ and the irregularities of the boundary $\partial \Omega$. (See esp. Theorem 2 and Remarks 3 and 4 below.) They are new and of (mathematical or physical) interest even when the weight function $r$ is positive. They enable one, for example, to study the vibrations of "drums with fractal boundary" ([L2-4], [LF1,2]) and variable mass density. Other significant physical applications include the study of flows through porous media and of the vibrations of cracked bodies.

"Indefinite elliptic problems" (i.e., involving an indefinite weight function) occur naturally by linearization of many semilinear elliptic equations and are of broad interest in applied mathematics, engineering, physics, and biology; for example, transport theory, hydrodynamics, crystal coloration, laser theory, reaction diffusion equations, .... . (See, e.g., [FL1,2] and the relevant references therein.) Recent mathematical works on these problems include [Be], [BS], [Fa1-3], [FF], [FL1-3], [He1-2], [HeKa], [KKZ], [KZ], [L1], [We].

The rest of this paper is organized as follows:

In Section 2, we consider the Dirichlet problem studied in [FL2, Section 3]. There we assume only that $\Omega_{ \pm}^{o}$, the interior of $\Omega_{ \pm}:=\{x \in \Omega / r(x) \gtrless 0\}$, is "Jordan contented" (without the hypothesis of "segment property") and recover and strengthen the asymptotic estimate of [FL2, Theorem 2, p. 339].

In Section 3, by use of "Whitney-type coverings" (with dyadic cubes which become smaller near the boundary), we obtain a result for the Dirichlet problem without any condition on $\Omega$. For the Neumann problem, we recover Theorem 2 of [FL2] (where an hypothesis was not made precise enough, as mentioned in [L2, Remark 3.5(d), p. 490] and [FL3], under some of the same conditions on $\Omega$ as in [FM, Mt1,2] where $r \equiv 1$.

The case when $\partial \Omega$ is "fractal" is also studied in Section 3 and hence, in the special case of the Dirichlet and Neumann Laplacians, this paper extends 
to indefinite weights some of the main results of [L2,3], esp. [L2, Theorems 2.1 and 4.1, pp. 479 and 510-511], where $r \equiv 1$. (See also [LF1,2] where a special case of this result is announced for the Dirichlet Laplacian.) The proof of our main result (Theorem 2 from Section 3) combines techniques from both [FL2] and [L2] (as well as [Mt2]) in order to deal with the oscillations and/or discontinuities of the (possibly) indefinite weight function, as well as with the roughness of the boundary.

\section{The Dirichlet PRoblem on a "JoRdAN CONTENTED SET"}

2.A. Hypotheses and results. $\left(H_{1}\right) \Omega$ is a nonempty bounded open set in $\mathbb{R}^{n}$ which is "Jordan contented"; the weight function $r$ belongs to $L^{\infty}(\Omega)$ and is allowed to change sign; furthermore $\left|\Omega_{+}^{o}\right|>0$, where $|\cdot|$ denotes the Lebesgue measure in $\mathbb{R}^{n}$ and $\Omega_{+}^{o}$ is the interior of $\Omega_{+}=\left\{x \in \mathbb{R}^{n} / r(x)>0\right\}$.

Remark 1. Recall that $\omega \subset \mathbb{R}^{n}$ is said to be "Jordan contented" (in French, "quarrable au sens de Jordan") if it can be well approximated from within and without by a finite union of dyadic cubes (see, e.g., [LoSt, Chapter 6, §§6-7] or [RS, p. 271]). In that case, its boundary $\partial \omega$ must have zero $n$-dimensional Lebesgue measure.

We now consider a covering of $\mathbb{R}^{n}$ by disjoint open cubes $\left(Q_{\zeta}\right)_{\zeta \in \mathbb{Z}^{n}}$ with side $\eta$ (so that $\mathbb{R}^{n}=\bigcup_{\zeta \in \mathbb{Z}^{n}} \overline{Q_{\zeta}}$ ) and we set

$$
\begin{gathered}
I\left(\Omega_{+}^{o}\right):=\left\{\zeta \in \mathbb{Z}^{n} / Q_{\zeta} \subset \Omega_{+}^{o}\right\}, \Omega_{+}^{\prime}=\left(\bigcup_{\zeta \in I\left(\Omega_{+}^{o}\right)} \overline{Q_{\zeta}}\right)^{o}, \\
J\left(\Omega_{+}^{o}\right):=\left\{\zeta \in \mathbb{Z}^{n} / Q_{\zeta} \cap \bar{\Omega} \neq \varnothing\right\},
\end{gathered}
$$

and

$$
\omega=\left(\bigcup_{\zeta \in J\left(\Omega_{+}^{o}\right) \backslash I\left(\Omega_{+}^{o}\right)} \overline{Q_{\zeta}}\right)^{o} .
$$

We next introduce two hypotheses (which were made in [FL2]):

$\left(H_{2}\right) \quad \Omega_{+}^{o}$ is "Jordan contented" and satisfies the " $\beta$-condition": given $\beta>$ 0 , there exist two positive constants $c_{0}$ and $\eta_{0}$ such that, for all $\eta \in\left(0, \eta_{0}\right]$,

$$
\frac{\#\left(J\left(\Omega_{+}^{o}\right) \backslash I\left(\Omega_{+}^{o}\right)\right)}{\#\left(I\left(\Omega_{+}^{o}\right)\right)} \leq c_{0} \eta^{\beta},
$$

where \#A denotes the number of elements in the finite set $A$.

$\left(H_{3}\right) \quad r_{+}$satisfies the " $\gamma$-condition on $\Omega_{+}^{o}$ ": given $\gamma \geq n$, there exists a positive constant $c_{1}$, which does not depend on $\zeta$, such that, for all $\eta$ small enough and for all $\zeta \in I\left(\Omega_{+}^{o}\right)$,

$$
\rho_{\zeta}(r):=\left\|r_{+}-r_{\zeta}\right\|_{L^{n / 2}\left(Q_{\zeta}\right)}^{n / 2} \leq c_{1} \eta^{\gamma},
$$

with $r_{\zeta} \geq 0$ defined by

$$
r_{\zeta}^{n / 2}:=\eta^{-n} \int_{Q_{\zeta}} r^{n / 2}=\eta^{-n}\|r\|_{L^{n / 2}\left(Q_{\zeta}\right)}^{n / 2}
$$

Moreover, $r_{+}$can be extended to a neighborhood of $\overline{\Omega_{+}}$to a positive bounded function, still denoted by $r_{+}$. Then 
Theorem 1. Under hypotheses $\left(H_{1}\right)$ to $\left(H_{3}\right)$, for all $\delta \in\left[\frac{1}{2(\nu+1)}, \frac{1}{2}\right]$, where $\nu:=\min (\beta, \gamma-n)$, we have

$$
R_{0}^{+}(\lambda ; r, \Omega)=O\left(\lambda^{\frac{n-1}{2}+\delta}\right) \text {, as } \lambda \rightarrow+\infty .
$$

This result is nothing else than Theorem 2, p. 339, in [FL2] for the Dirichlet problem, but with weaker hypotheses. An analogous result could of course be obtained for negative eigenvalues under analogous hypotheses on $\Omega_{-}$and $r_{-}$. The exponent $\frac{n-1}{2}+\delta$ depends on the regularity of the weight function $r$ (the larger $\gamma$, the "smoother" $r$ ) and of the boundary $\partial \Omega$ (the larger $\beta$, the "smoother" $\partial \Omega$ ) so that for smooth data, this exponent is as close as we want from $\frac{n-1}{2}$, the best possible exponent.

2.B. Proof of Theorem 1. We first note that for all $\rho>0$,

$$
N_{0}^{+}\left(\lambda ; r, \Omega_{+}^{o}\right) \leq N_{0}^{+}(\lambda ; r, \Omega) \leq N_{0}^{+}\left(\lambda ; r_{+}+\rho, \Omega\right) .
$$

Hence, by letting $\rho$ tend to zero, exactly as in [L1], [FL1], .., Theorem 1 when $r$ changes sign can be derived from the case when $r$ is positive.

Thus from now on in this section, we assume that

$\left(H_{4}\right) r$ is positive, so that $\Omega=\Omega_{+}^{0}$ and $N_{0}^{-}(\lambda ; r, \Omega)=0$;

in that case, we write in short

$$
N_{i}(\lambda ; r, \Omega):=N_{i}^{+}(\lambda ; r, \Omega)
$$

and

$$
I:=I\left(\Omega_{+}^{o}\right), J:=J\left(\Omega_{+}^{o}\right) .
$$

The monotonicity of $N_{0}(\lambda ; r, \Omega)$ with respect to $\Omega$ allows us to consider the problem on $\left(\overline{\Omega^{\prime}} \cup \bar{\omega}\right)^{o}$ which is larger than $\Omega$; moreover, by use of the method of the Dirichlet-Neumann bracketing [CH, RS, Mt1, ..., FL1-2, L1-2, ... ], we have

$N_{0}\left(\lambda ; r, \Omega^{\prime}\right) \leq N_{0}(\lambda ; r, \Omega) \leq N_{0}\left(\lambda ; r,\left(\overline{\Omega^{\prime}} \cup \bar{\omega}\right)^{o}\right) \leq N_{1}\left(\lambda ; r, \Omega^{\prime}\right)+N_{1}(\lambda ; r, \omega)$.

Hence, by subtracting the "Weyl term" $W(\lambda ; r, \Omega)$, we get

$$
A_{0} \leq R_{0}(\lambda ; r, \Omega) \leq A_{1}+A_{2},
$$

where for $i=0$ or 1 ,

$$
A_{i}:=\sum_{\zeta \in I} R_{i}\left(\lambda ; r_{\zeta}, Q_{\zeta}\right)
$$

and

$$
A_{2}:=N_{1}(\lambda ; r, \omega)-W\left(\lambda ; r, \Omega \backslash \overline{\Omega^{\prime}}\right) \leq N_{1}(\lambda ; r, \omega) .
$$

The "interior terms" $A_{0}$ and $A_{1}$ can be handled exactly as the term $A$ defined by Eq. (19.0) in [FL2], p. 342. (Note that in [FL2], the dimension is denoted by $k$ instead of $n$.) Hence, for a given $\lambda>0$ large enough, we choose (as in [FL2, Eq. (24), p. 343]):

$$
\eta=\lambda^{-a} \text { with } a \in(0, \delta]
$$

and we deduce that there exists $c>0$ such that the counterpart of Eq. (36) in [FL2, p. 345] holds:

$$
R_{0}(\lambda ; r, \Omega) \geq-c \lambda^{\frac{n-1}{2}+\delta}
$$


In the same manner, we obtain an upper bound for $A_{1}$, which follows from Eqs. (38) to (40) in [FL2, p. 346]:

$$
\left|A_{1}\right| \leq c \lambda^{\frac{n-1}{2}+\delta} \text {. }
$$

For the "boundary term" $N_{1}(\lambda ; r, \omega)$ which appears in $(2.7)$, we again use the Dirichlet-Neumann bracketing and the monotonicity of $N_{1}(\lambda ; r, \omega)$ with respect to $r$ :

$$
\begin{aligned}
A_{2} & \leq N_{1}(\lambda ; r, \omega) \leq N_{1}(\lambda ; M, \omega)=N_{1}(\lambda M ; 1, \omega) \\
& \leq \sum_{\zeta \in J \backslash I} N_{1}\left(\lambda M ; 1, Q_{\zeta}\right) \leq c(\#(J \backslash I)) \eta^{n} \lambda^{n / 2},
\end{aligned}
$$

where $M<\infty$ is an upper bound for $r_{+}$; note that $M$ exists by hypothesis $\left(H_{3}\right)$. The last inequality is well known since the problem corresponding to $N_{1}\left(\lambda ; 1, Q_{\zeta}\right)$ is the Laplacian on a cube "without weight", i.e., with $r \equiv 1$.

By use of $\left(H_{1}\right),\left(H_{2}\right),(2.4)$, and (2.8), there exists $c>0$ such that

$$
\#(J \backslash I) \leq c \lambda^{a(n-\beta)} \text { with } a \in(0, \delta] .
$$

Hence, combining (2.11) with (2.12), we obtain as in [FL2, Eq. (28), p. 344]:

$$
A_{2} \leq c \lambda^{\frac{n}{2}-a \beta} \leq c \lambda^{\frac{n-1}{2}+\delta},
$$

provided that we choose a positive number $a$ such that

$$
\frac{1}{\nu}\left(\frac{1}{2}-\delta\right) \leq a \leq \delta
$$

Note that this choice of $a$ is possible since by hypothesis of Theorem 1,

$$
\frac{1}{2(\nu+1)} \leq \delta \leq \frac{1}{2}
$$

Theorem 1 for $r>0$ follows from (2.5), (2.9), (2.10), and (2.13).

\section{IRREGULAR BOUNDARIES}

3.A. Introduction. In the previous section, the upper bound for $N_{0}(\lambda ; r, \Omega)$ was very simple to establish since by use of the monotonicity of $N_{0}(\lambda ; r, \Omega)$ with respect to $\Omega$, we could include $\Omega$ in a (finite) union of cubes. But this is not possible for the Neumann problem. Hence for obtaining more precise estimates at the boundary, we consider a covering of $\mathbb{R}^{n}$ by dyadic cubes. This partition is used in particular in [CH] and by many authors (e.g., [Mt2], [L2], ... ); it is sometimes referred to as a "Whitney-type covering".

Let $O$ be an open set in $\mathbb{R}^{n}$. For a given $\eta_{0}>0$, we consider a covering of $\mathbb{R}^{n}$ by disjoint open cubes $\left(Q_{\zeta_{q}}\right)_{\zeta_{q} \in \mathbf{Z}^{n}}$ with side

$$
\eta_{q}=\eta_{0} 2^{-q}, q \in \mathbb{N} \text {. }
$$

Set

$$
I_{0}(O)=\left\{\zeta_{0} \in \mathbb{Z}^{n} / Q_{\zeta_{0}} \subset O\right\} ; O_{0}^{\prime}=\left(\bigcup_{\zeta_{0} \in I_{0}(O)} \overline{Q_{\zeta_{0}}}\right)^{o}
$$


and

$$
I_{q}(O)=\left\{\zeta_{q} \in \mathbb{Z}^{n} / Q_{\zeta_{q}} \subset O_{q-1}^{\prime \prime}\right\} ; O_{q}^{\prime}=\left(\overline{O_{q-1}^{\prime}} \cup\left(\bigcup_{\zeta_{q} \in I q(O)} \overline{Q_{\zeta_{q}}}\right)\right)^{o}
$$

and

$$
O_{q}^{\prime \prime}=O \backslash \bar{O}_{q}^{\prime}, \text { for } q \geq 1 .
$$

We now weaken the " $\beta$-condition" which implies that $\Omega_{+}^{o}$ is a Jordan contented set; this enables us to deal with more irregular boundaries and even fractal boundaries, as in [L2-3].

3.B. Hypotheses and results. $\left(H_{1}^{\prime}\right) \Omega$ is a (nonempty) bounded open set in $\mathbb{R}^{n}$ with $n \geq 1$; the weight function $r$ belongs to $L^{\infty}(\Omega)$ and is allowed to change sign; furthermore, $\left|\Omega_{+}^{o}\right|>0$.

$\left(H_{2}^{\prime}\right) \quad \Omega_{+}^{o}$ is such that there exists $d \in[n-1 ; n)$ satisfying

$$
\mathscr{M}^{*}\left(d ; \partial \Omega_{+}^{o}\right):=\lim _{\epsilon \rightarrow 0+} \sup ^{-(n-d)}\left|\tilde{\Gamma}_{\epsilon}\right|<\infty,
$$

with

$$
\tilde{\Gamma}_{\epsilon}:=\Gamma_{\epsilon} \cap \Omega_{+}^{o} \text { and } \Gamma_{\epsilon}:=\left\{x \in \mathbb{R}^{n} / \operatorname{dist}\left(x, \partial\left(\Omega_{+}^{o}\right)\right)<\epsilon\right\} ;
$$

here, dist $\left(\right.$, .) denotes the Euclidean distance in $\mathbb{R}^{n}$.

$\left(H_{3}^{\prime}\right) r_{+}$satisfies the " $\gamma$-condition" on $\Omega_{+}^{o}$ (adapted to the covering $Q_{\zeta_{q}}$ ) with $\gamma>d$; i.e., there exists $c_{2}>0$ which does not depend on $\zeta_{q} \in I_{q}\left(\Omega_{+}^{o}\right)$ and there exists $\eta_{1}>0$ such that for all $\eta \in\left(0, \eta_{1}\right]$, for all $q \in \mathbb{N}$,

$$
\rho_{\zeta}(r) \leq c_{2} \eta_{q}^{\gamma}
$$

where $\rho_{\zeta}$ is defined as in $(2.2 \mathrm{a})$.

$\left(H_{4}^{\prime}\right)$ For the Neumann problem, we assume that $\left(H_{2}^{\prime}\right)$ holds with $\tilde{\Gamma}_{\epsilon}$ replaced by $\Gamma_{\epsilon}$; we assume in addition that $\Omega_{+}^{o}$ satisfies either the "extension property" (there exists a continuous linear extension of $H^{1}(\Omega)$ onto $H^{1}\left(\mathbb{R}^{n}\right)$, e.g., [L2, Definition 4.3, p. 510]) or the " $\left(C^{\prime}\right)$ condition" ([Mt2, pp. 154-156]: there exists a finite covering of $\partial \Omega$ by open sets $\left(O_{j}\right)_{j=1, \ldots, J_{0}^{\prime}}$, with $O_{j} \subset \mathbb{R}^{n}$; there are open sets $U_{j}^{\prime}$ in $\mathbb{R}^{n-1}$, positive numbers $h_{j}$, upper semi-continuous functions $\varphi_{j}:\left(-h_{j}, 2 h_{j}\right) \rightarrow U_{j}^{\prime}$, and $C^{1}$ diffeomorphisms $\theta_{j}: O_{j} \cap \Omega \rightarrow V_{j}$ with

$$
V_{j}=\left\{\xi=\left(\xi_{1}, \xi^{\prime}\right) \in \mathbb{R}^{n} / \xi^{\prime} \in U_{j}^{\prime} ; \varphi_{j}\left(\xi^{\prime}\right)<\xi_{1}<2 h_{j}\right\} .
$$

Furthermore, for all $x$ and $y$ in $O_{j} \cap \Omega$, there exists $s>0$ such that the path $\mathscr{L}_{s}(x, y)$ associated to the reunion of segments

$$
\left[\theta_{j}(x), \theta_{j}(x)+s e_{1}\right] \cup\left[\theta_{j}(x)+s e_{1}, \theta_{j}(y)+s e_{1}\right] \cup\left[\theta_{j}(y)+s e_{1}, \theta_{j}(y)\right]
$$

lies within $O_{j} \cap \Omega$ (here, $e_{1}$ denotes the first vector of the standard basis in $\left.\mathbb{R}^{n}\right)$. Moreover, if we denote by $\rho_{j}(x, y)$ the infimum of the lengths of all such $\mathscr{L}_{s}(x, y)$ in $O_{j} \cap \Omega$, there exists $k_{0}$ such that for all $j \in\left\{1,2, \ldots, J_{0}\right\}$,

$$
\rho_{j}(x, y) \leq k_{0} \operatorname{dist}(x, y) .
$$

Remark 2. (a) If the open set $\omega$ satisfies the "segment property" [Ag, p. 11], then it satisfies the " $\left(C^{\prime}\right)$ condition"; this is the case, for example, if $\partial \omega$ is 
Lipschitz. The “ $\left(C^{\prime}\right)$ condition" was first introduced in [FM, p. 914, §3], and then used in [Mt1-2], [FL1-2], [L1-4]. Roughly, it means that the boundary $\partial \Omega$ is not "too long": the Euclidean distance dist $(x, y)$ is equivalent to $d_{\Omega}(x, y)$, the minimal length of continuous paths from $x$ to $y$ within $\Omega$. Note that if the " $\left(C^{\prime}\right)$ condition" is not satisfied, then the usual Weyl asymptotic formula (1.1) may fail for the Neumann problem. (See, e.g., the counterexamples when $i=1$ and $r \equiv 1$ in [FM] and [Mt2, §VII.1, pp. 200-204].)

(b) When $n=2$, the simply connected domain $\omega$ satisfies the "extension property" if and only if it is a quasidisk, i.e., the image of the unit disk under a quasiconformal mapping. (See, e.g., [L2, Example 4.2, p. 510] and the references therein.) The boundary $\partial \omega$ is then a quasicircle (roughly, a distorted circle) and may be extremely irregular [L2, p. 510].

(c) Intuitively, the more "regular" the boundary $\partial \Omega_{+}^{\circ}$, the smaller we may choose $d$ in hypothesis $\left(H_{2}^{\prime}\right)$. Similarly, the more "regular" the weight function $r$, the larger we may choose $\gamma$ in hypothesis $\left(H_{3}^{\prime}\right)$. For example, if $r \equiv 1$, as in [L2], then clearly, any $\gamma>0$ is suitable. Further, it follows from (the argument provided in) [FL2, Example 2, p. 333] that if $r$ is Hölder continuous of order $\theta \in(0,1)$ and bounded away from zero on $\Omega_{+}^{\circ}$, then it satisfies the " $\gamma$-condition" on $\Omega_{+}^{\circ}$ with $\gamma=n+(n \theta / 2)$; in particular, $\gamma>n$ in that case. Theorem 2. Under the above hypotheses, we have for $i=0$ or 1:

(i) If $d \in(n-1, n)$ (the "fractal case")

$$
R_{i}^{+}(\lambda ; r, \Omega):=N_{i}^{+}(\lambda ; r, \Omega)-W\left(\lambda ; r_{+}, \Omega\right)=O\left(\lambda^{t / 2}\right), \text { as } \lambda \rightarrow+\infty,
$$

where $t:=\max (d, d+n-\gamma)$. [Hence $t=d$ if $\gamma \geq n$ and $t=d+n-\gamma$ if $\gamma<n$.]

(ii) If $d=n-1$ (the "nonfractal case"),

$$
R_{i}^{+}(\lambda ; r, \Omega)= \begin{cases}O\left(\lambda^{\frac{n-1}{2}} \log \lambda\right) & \text { when } \gamma>n, \\ O\left(\lambda^{\frac{n-1}{2}}+\frac{n-\gamma}{2}\right) & \text { when } \gamma \leq n\end{cases}
$$

Theorem 2 extends to problems with indefinite weights earlier results obtained for $r \equiv 1$ ([Mt1-2], [L2-3]). It also makes more precise and extends to irregular boundaries results of [FL2] where an hypothesis needed to be completed (see [L2, Remark 3.5(d), p. 490]).

Remark 3. (a) Hypothesis $\left(H_{2}^{\prime}\right)$ which replaces in this section the " $\beta$-condition" says that the (interior Bouligand-) Minkowski dimension $D$ of $\partial \Omega_{+}^{0}$ is $\leq d$. (See, e.g., [L2, Definition 2.1(b), pp. 474-475 and §3]. For relationships between the " $\beta$-condition" and the Minkowski dimension, see [L2, Corollary 3.3 and Remark 3.5, pp. 489-490].) When the weight $r$ is "smooth enough" (compared with $\partial \Omega$ ), viz $\gamma \geq n$, then $t=d$ and we recover for $d>n-1$ results of [L2-3] in the special case of the (Dirichlet or Neumann) Laplacian. (See Remark 2(c) above and [L2, Theorem 2.3, pp. 482-483].) Indeed, the irregularity (fractality) of the boundary of $\Omega_{+}^{o}$ can be due to that of $\Omega\left(\partial \Omega \cap \partial\left(\Omega_{+}^{o}\right)\right)$ or that of $r$ $\left(\partial\left(\Omega_{+}^{o}\right) \backslash \partial \Omega\right): d<\gamma<n$.

(b) The use of the Minkowski dimension $D$ for rough boundaries (in a related context) was first made by Brossard and Carmona in [BrCa]. In [BrCa], the authors also obtained (pre-Tauberian) error estimates (expressed in terms of $D$ ) for the short time asymptotics of the "partition function" $Z(t)=$ Trace $\left(e^{t \Delta}\right)$, when $\Delta$ is the Dirichlet Laplacian and $r \equiv 1$. 
(c) Strictly speaking and to be in keeping with the terminology used in [L24], the "fractal" (resp., "nonfractal") case is that when $D \neq n-1$ (resp., $D=$ $n-1)$. Recall from [L2] that we always have $D \in[n-1, n]$ and that if $0<\mathscr{M}^{*}\left(d ; \partial \Omega_{+}^{o}\right)<\infty$, then $d=D$, the (interior) Minkowski dimension of $\partial \Omega_{+}^{o}$.

Of course, an analogous theorem holds for $N_{i}^{-}(\lambda ; r, \Omega)$ under analogous hypotheses.

As in the previous section, we will prove Theorem 2 for $r>0$; the case where $r$ changes sign can be derived as indicated above. Therefore, from now on, we assume that

$\left(H_{5}^{\prime}\right) r$ is positive, hence $\Omega=\Omega_{+}^{o}$, and as above we set

$$
N_{i}(\lambda ; r, \Omega)=N_{i}^{+}(\lambda ; r, \Omega) \text {. }
$$

We also let, in view of (3.3),

$$
I_{q}:=I_{q}(\Omega)
$$

and

$$
\omega_{q}:=\Omega_{q}^{\prime \prime} \text {. }
$$

3.C. A lower bound. We establish here a lower bound for $R_{i}(\lambda ; r, \Omega)=$ $N_{i}(\lambda ; r, \Omega)-W(\lambda ; r, \Omega), i=0$ or 1 , when $r$ is positive. The numbers $\gamma$ and $d$ are given, with $\gamma>d$. Choose $\lambda>1$ and then $\eta_{0}=\lambda^{-a}$ where $a>\frac{n-d}{2(\gamma-d)}$ with $\eta_{0}$ small enough so that $\left(H_{2}^{\prime}\right)$ or $\left(H_{4}^{\prime}\right)$ for the Dirichlet or Neumann problem, respectively, implies that there exists $c_{3}>0$ such that for all $q \in \mathbb{N}$,

$$
\left(\#\left(I_{q}\right)\right) \eta_{q}^{n} \leq c_{3} \eta_{q}^{n-d},
$$

where $\eta_{q}$ is defined by (3.1) and $I_{q}$ by (3.3) and (3.5a).

Since $r$ is bounded, there exists $M>0$ such that for almost all $x \in$ $\Omega,|r(x)| \leq M$, and hence, $\lambda$ being given, there exists $P \in \mathbb{N}$ such that, for all $q>P$,

$$
N_{0}\left(\lambda ; r, Q_{\zeta_{q}}\right)=0
$$

We define

$$
P:=\max \left\{q \in \mathbb{N} / N_{0}\left(\lambda ; M, Q_{\zeta_{q}}\right) \neq 0\right\} .
$$

Note that the integer $P$ depends on $\lambda$. By means of (3.3) and the usual inequalities on $N_{i}(\lambda ; r, \Omega)$, we can write:

$$
\begin{aligned}
N_{1}(\lambda ; r, \Omega) & \geq N_{0}(\lambda ; r, \Omega) \geq N_{0}\left(\lambda ; r, \Omega_{P}^{\prime}\right) \\
& \geq \sum_{q=0}^{P} \sum_{\zeta_{q} \in I_{q}} N_{0}\left(\lambda ; r, Q_{\zeta_{q}}\right) .
\end{aligned}
$$

Then, by subtracting the "Weyl term", we obtain

$$
R_{i}(\lambda ; r, \Omega)=N_{i}(\lambda ; r, \Omega)-W(\lambda ; r, \Omega) \geq A_{1}+A_{2}+A_{3}+A_{4},
$$


with

$$
A_{1}:=\sum_{q=0}^{P} \sum_{\zeta_{q} \in I q}\left(N_{0}\left(\lambda ; r, Q_{\zeta_{q}}\right)-N_{0}\left(\lambda ; r_{\zeta_{q}}, Q_{\zeta_{q}}\right)\right)
$$

$$
A_{2}:=\sum_{q=0}^{P} \sum_{\zeta_{q} \in I q} R\left(\lambda ; r_{\zeta_{q}}, Q_{\zeta_{q}}\right)
$$

$$
A_{3}:=\sum_{q=0}^{P} \sum_{\zeta_{q} \in I q}\left(W\left(\lambda ; r_{\zeta_{q}}, Q_{\zeta_{q}}\right)-W\left(\lambda ; r, Q_{\zeta_{q}}\right)\right)
$$

and

$$
A_{4}:=-W\left(\lambda ; r, \omega_{P}\right) .
$$

We first note that, with $\rho_{\zeta}$ defined as in $(2.2 \mathrm{a})$,

$$
\left|N_{i}\left(\lambda ; r, Q_{\zeta}\right)-N_{i}\left(\lambda ; r_{\zeta}, Q_{\zeta}\right)\right| \leq N_{i}\left(\lambda ;\left|r-r_{\zeta}\right|, Q_{\zeta}\right) \leq \rho_{\zeta}(r) \lambda^{n / 2} .
$$

Therefore, by $\left(H_{3}^{\prime}\right),(3.1),(3.6)$, and $(3.7)$, we have

$$
\begin{aligned}
-A_{1} & \leq c \sum_{q=0}^{P}\left(\#\left(I_{q}\right)\right) \eta_{q}^{\gamma} \lambda^{n / 2} \leq c \lambda^{n / 2} \sum_{q=0}^{P} \eta_{q}^{\gamma-d} \\
& \leq c \lambda^{n / 2} \eta_{0}^{\gamma-d} \sum_{q=0}^{P} 2^{-q} \leq c^{\prime} \lambda^{(n / 2)-a(\gamma-d)} .
\end{aligned}
$$

We note that, when $d<\gamma \leq n$, we have $\frac{n-d}{\gamma-d}>1$ and thus our choice of $a$ preceding Eq. (3.6) implies $a>\frac{1}{2}$. Thus, for $d \in[n-1, n)$,

$$
-A_{1} \leq c \lambda^{t / 2}, \quad \text { with } t=\max (d, d+n-\gamma) .
$$

We now consider $A_{2}$ (which is negative); it can be handled as in [FL2, Eq. (21), p. 342]: we know from [CH, Section 6.4] (or [RS, Proposition 2, pp. 266-267]) that there exists $c_{0}^{\prime}>0$ such that, for $i=0$ or 1 , for all $\wedge \geq 1$ and all $q \in \mathbb{N}$,

$$
\left|R_{i}\left(\wedge ; 1, Q_{\zeta_{q}}\right)\right| \leq c_{0}^{\prime}\left[1+\left(\wedge \eta_{q}^{2}\right)^{(n-1) / 2}\right]
$$

Consequently,

$$
\left|A_{2}\right| \leq c_{0}^{\prime} \sum_{q=0}^{P}\left(\#\left(I_{q}\right)\right)\left[1+\left(\lambda r_{\zeta} \eta_{q}^{2}\right)^{(n-1) / 2}\right] .
$$

We deduce from (3.8) that there exists $c_{4}>0$ satisfying

$$
\frac{1}{2} c_{4} \sqrt{\lambda}<2^{P}<c_{4} \sqrt{\lambda} .
$$

Hence, by combining (3.1), (3.6), (3.15), and (3.16), and since $r$ is bounded by $M$, we obtain

$$
\left|A_{2}\right| \leq c^{\prime} \sum_{q=0}^{P} \eta_{q}^{-d}+c^{\prime \prime} \lambda^{(n-1) / 2} \sum_{q=0}^{P} \eta_{q}^{n-1-d} .
$$


Therefore,

$$
\begin{gathered}
\left|A_{2}\right| \leq c \lambda^{d / 2} \text { for } d>n-1, \\
\left|A_{2}\right| \leq c \lambda^{(n-1) / 2} \ln \lambda \text { for } d=n-1 .
\end{gathered}
$$

By definition of $r_{\zeta_{q}}$ in (2.2), it is obvious in view of $(3.10 \mathrm{c})$ that

$$
A_{3}=0 \text {. }
$$

Finally we deduce from $(3.16),\left(H_{1}^{\prime}\right)$, and $\left(H_{2}^{\prime}\right)$ or $\left(H_{4}^{\prime}\right)$ that

$$
\left|A_{4}\right|=W\left(\lambda ; r, \omega_{P}\right)=c \int_{\omega_{P}}(\lambda r)^{n / 2} \leq c \lambda^{n / 2}\left|\omega_{P}\right| \leq c \lambda^{n / 2} \eta_{P}^{n-d} \leq c \lambda^{d / 2} .
$$

Hence it follows from (3.10), (3.13), and (3.17) to (3.19) that, for $r$ regular enough, i.e., for $\gamma>d$, we have:

(i) When $d=n-1$,

$$
\begin{aligned}
R_{i}(\lambda ; r, \Omega) & \geq-c \lambda^{\frac{n-1}{2}} \ln \lambda \text { if } \gamma>n \\
& \geq-c \lambda^{\frac{n-1}{2}+\frac{n-\gamma}{2}} \quad \text { if } d<\gamma \leq n .
\end{aligned}
$$

(ii) When $d \in(n-1, n)$,

$$
R_{i}(\lambda ; r, \Omega) \geq-c \lambda^{t / 2}
$$

where

$$
t=\max (d, d+n-\gamma) \text {. }
$$

Remark 4. (a) In particular, when $r \equiv 1$, we have $\gamma \geq n$ [see Remark 2(c)]; if $d \in(n-1, n)$, we then recover, in the special case of the Laplacian, the estimate $R_{i}(\lambda ; r, \Omega) \geq-c \lambda^{d / 2}$ which has been established in [L2, Theorem 2.3 , p. 482] and [L3]. As was shown in [L2], [L4], this estimate is sharp for every $d \in(n-1, n)$ and for $i=0$ or 1 .

(b) Note that when $r$ is Hölder continuous on $\Omega$ and bounded away from zero, then $\gamma>n$ (by Remark 2(c) above) and hence $\gamma>d$ is automatically fulfilled.

(c) When $n=1$ and $0<d<1$, the lower bound is of the form $-c \lambda^{d / 2} \ln \lambda$ [resp. $-c \lambda^{d / 2}$ ] when $\gamma>1$ [resp. $\leq 1$ ].

3.D. Upper bound. For the Dirichlet problem, we can simply include $\Omega$ in a finite union of cubes; up to a set with Lebesgue measure zero, we have

$$
\Omega \subset O_{P}:=\bigcup_{q=0}^{P} \bigcup_{\zeta_{q} \in I q} Q_{\zeta_{q}} \cup\left(\bigcup_{\zeta_{P} \in I_{P}^{\prime}} Q_{\zeta_{P}}\right)
$$

where

$$
I_{P}^{\prime}=\left\{\zeta_{P} \in \mathbb{Z}^{n} / Q_{\zeta_{P}} \cap \partial \Omega \neq \varnothing\right\} .
$$

We note that as for $I_{q}$ in (3.6), we have

$$
\#\left(I_{P}^{\prime}\right) \leq c_{3} \eta_{P}^{-d} .
$$

Therefore, we can write

$$
N_{0}(\lambda ; r, \Omega) \leq N_{0}\left(\lambda ; r, O_{P}\right) \leq \sum_{q=0}^{P} \sum_{\zeta_{q} \in I q} N_{1}\left(\lambda ; r, Q_{\zeta_{q}}\right)+\sum_{\zeta_{P} \in I_{P}^{\prime}} N_{1}\left(\lambda ; r, Q_{\zeta_{P}}\right)
$$


In (3.23), in order to extend $r$, we must assume $\lambda$ (and hence $P$ ) large enough.

By subtracting the Weyl term, we obtain

$R_{0}(\lambda ; r, \Omega) \leq \sum_{q=0}^{P} \sum_{\zeta_{q} \in I q} R_{1}\left(\lambda ; r, Q_{\zeta_{q}}\right)+\sum_{\zeta_{P} \in I_{P}^{\prime}} N_{1}\left(\lambda ; r, Q_{\zeta_{P}}\right) \leq B_{1}+B_{2}+B_{3}+B_{4}$,

where

$$
\begin{aligned}
B_{1} & :=\sum_{q=0}^{P} \sum_{\zeta_{q} \in I q}\left(N_{1}\left(\lambda ; r, Q_{\zeta_{q}}\right)-N_{1}\left(\lambda ; r_{\zeta_{q}}, Q_{\zeta_{q}}\right)\right), \\
B_{2} & :=\sum_{q=0}^{P} \sum_{\zeta_{q} \in I q}\left(N_{1}\left(\lambda ; r_{\zeta_{q}}, Q_{\zeta_{q}}\right)-W\left(\lambda ; r_{\zeta_{q}}, Q_{\zeta_{q}}\right)\right), \\
B_{3} & :=\sum_{q=0}^{P} \sum_{\zeta_{q} \in I q}\left(W\left(\lambda ; r_{\zeta_{q}}, Q_{\zeta_{q}}\right)-W\left(\lambda ; r, Q_{\zeta_{q}}\right)\right),
\end{aligned}
$$

and

$$
B_{4}:=\sum_{\zeta_{P} \in I_{P}^{\prime}} N_{1}\left(\lambda ; r, Q_{\zeta_{P}}\right) .
$$

The first three terms $B_{1}, B_{2}, B_{3}$ can be handled exactly as $A_{1}, A_{2}, A_{3}$ in the previous section (§3.C), and we obtain:

$$
\begin{aligned}
& B_{1} \leq c \lambda^{t / 2} \text { with } t=\max (d, d+n-\gamma), \\
& B_{2} \leq c \lambda^{d / 2} \text { for } d>n-1, \\
& B_{2} \leq c \lambda^{(n-1) / 2} \ln \lambda \text { when } d=n-1, \\
& B_{3}=0 .
\end{aligned}
$$

Since $r$ is bounded, we have

$$
B_{4} \leq \sum_{\zeta_{P} \in I_{P}^{\prime}} N_{1}\left(\lambda M ; 1, Q_{\zeta_{P}}\right) \leq c \lambda^{n / 2} \eta_{P}^{n}\left(\#\left(I_{P}^{\prime}\right)\right)
$$

so that, by (3.1), (3.16), and (3.22),

$$
B_{4} \leq c \lambda^{d / 2} .
$$

Hence, by combining (3.26) to (3.29) with (3.24), we have for $\gamma>d$, (3.30)

$$
\begin{aligned}
R_{0}(\lambda ; r, \Omega) & \leq c \lambda^{\frac{n-1}{2}} \ln \lambda \text { when } d=n-1 \text { and } \gamma>n, \\
& \leq c \lambda^{\frac{n-1}{2}+\frac{n-\gamma}{2}} \text { when } d=n-1 \text { and } d<\gamma \leq n, \\
& \leq c \lambda^{t / 2} \text { with } t=\max (d, d+n-\gamma), \text { when } d \in(n-1, n) .
\end{aligned}
$$

For the Neumann problem, we cannot write (3.23); we simply have by means of definition $(3.5 b)$ :

$$
R_{1}(\lambda ; r, \Omega) \leq B_{1}+B_{2}+B_{3}+B_{4}^{\prime},
$$

where $B_{1}, B_{2}, B_{3}$ are given by (3.25a-c) and

$$
B_{4}^{\prime}:=N_{1}\left(\lambda ; r, \omega_{P}\right) \text {. }
$$


Since $r$ is bounded,

$$
B_{4}^{\prime} \leq N_{1}\left(\lambda M ; 1, \omega_{P}\right) .
$$

We now have to study a Neumann problem (without weight function) on the boundary strip $\omega_{P}$. We deal as in [FM], [Mt1-2] or [L2, pp. 496-497]. We denote by $\tilde{u}$ the extension of $u$ to $\mathbb{R}^{n}$ with $\tilde{u} \in H^{1}\left(\mathbb{R}^{n}\right)$. On each cube $Q_{\zeta_{q}}$ we approximate $\tilde{u}$ by its mean value $\overline{u_{\zeta_{q}}}$. Set

$$
v:=\sum_{\zeta_{P} \in I_{P}^{\prime}} \overline{u_{\zeta_{P}}} \mathbb{1}_{Q_{\zeta_{P}}},
$$

where

$$
\mathbb{1}_{Q_{\zeta_{P}}}(x):= \begin{cases}1 & \text { when } x \in Q_{\zeta_{P}} \\ 0 & \text { when } x \notin Q_{\zeta_{P}} .\end{cases}
$$

By use of inequality (1) in [FM, p. 915] or inequality 5.2 in [Mt1], we obtain

$$
\|u-v\|_{L^{2}\left(\omega_{P}\right)}^{2} \leq c \eta_{P}^{2}\|u\|_{H^{1}(\Omega)}^{2} .
$$

(See also [L2].) We note that $v$ defined by (3.34) lies in a (\#( $\left.\left.I_{P}^{\prime}\right)\right)$-dimensional subspace of $H^{1}\left(\mathbb{R}^{n}\right)$. Hence in view of (3.22) and (3.35):

$$
B_{4}^{\prime} \leq c \lambda^{d / 2} \text {. }
$$

It follows from (3.26) to (3.28) combined with (3.31) and (3.36) that the counterpart of (3.30) also holds for $R_{1}(\lambda ; r, \Omega)$ [instead of $R_{0}(\lambda ; r, \Omega)$ ], and hence Theorem 2 is proved.

Remark 5. (a) Our results (Theorems 1 and 2) could be extended to more general elliptic operators, much as in [Mt1-2], [FL2], and [L2-3], although we chose not do so in order to keep our arguments reasonably short and simple.

(b) It would be interesting to investigate whether, under suitable hypotheses, the results of [LP1,2]-that establish when $n=1$ (and $r \equiv 1$ ) the "modified Weyl-Berry conjecture" of [L2] concerning the existence of a (monotonic) asymptotic second term for $N(\lambda)$-can be extended to "fractal strings" ([L2], [L4], [LP1-2]) with variable mass density.

\section{ACKNOWLEDGMENTS}

The authors wish to thank the University of Montreal (Canada) and the University College in Cardiff, Wales (G.B.). J. F. thanks the Universities of Athens (Ohio) and Rutgers, USA, and M. L. L. is grateful to the Department of Mathematics of Yale University, where he was Visiting Professor while part of this paper was completed.

\section{REFERENCES}

[Ag] S. Agmon, Lectures on elliptic boundary values problem, Van Nostrand, Princeton, NJ, 1965.

[Be] R. Beals, Indefinite Sturm-Liouville problems and half-range completeness, J. Differential Equations 56 (1985), 391-407.

[BS] M. S. Birman and M. Z. Solomjak, Quantitative analysis in Sobolev imbeddings theorems and applications to spectral theory, Amer. Math. Soc. Transl. Ser. 2, vol. 114, Amer. Math. Soc., Providence, RI, 1980.

[BrCa] J. Brossard and R. Carmona, Can one hear the dimension of a fractal?, Comm. Math. Phys. 104 (1986), 103-122. 
[CH] R. Courant and D. Hilbert, Methods of mathematical physics, Vol. I, Interscience, New York, 1953.

[Fa1] M. Faierman, Elliptic problems involving an indefinite weight, Trans. Amer. Math. Soc. 320 (1990), 253-279.

[Fa2] _ Non-selfadjoint elliptic problems involving an indefinite weight, Comm. Partial Differential Equations 15 (1990), 939-982.

[Fa3] __ Eigenfunction expansions for a non-selfadjoint elliptic boundary value problem involving an indefinite weight, preprint, Dept. of Math., Univ. of the Witwatersrand, Johannesburg, South Africa (to appear).

[FF] J. Fleckinger and M. El Fetnassi, Comportement asymptotique des valeurs propres de problèmes elliptiques "non définis à droite", C. R. Acad. Sci. Paris Sér. I Math. 299 (1984), 599-602.

[FL1] J. Fleckinger and M. L. Lapidus, Eigenvalues of elliptic boundary value problems with an indefinite weight function, Trans. Amer. Math. Soc. 295 (1986), 305-324.

[FL2] __ Remainder estimates for the asymptotics of elliptic eigenvalue problems with indefinite weights, Arch. Rational Mech. Anal. 98 (1987), 323-356.

[FL3] _ Schrödinger operators with indefinite weight functions: asymptotics of eigenvalues with remainder estimates, Differential Integral Equations 7 (1994), 1389-1418.

[FM] J. Fleckinger and G. Métivier, Théorie spectrale des opérateurs uniformément elliptiques sur quelques ouverts irréguliers, C. R. Acad. Sci. Paris Sér. A 276 (1973), 913-916.

[He1] P. Hess, On the asymptotic distribution of eigenvalues of some nonselfadjoint problems, Bull. London Math. Soc. 18 (1986), 181-184.

[He2] _ On the spectrum of elliptic operators with indefinite weights, Linear Algebra Appl. 84 (1986), 99-109.

[HeKa] P. Hess and T. Kato, On some linear and nonlinear eigenvalue problems with an indefinite weight function, Comm. Partial Differential Equations 5 (1980), 999-1030.

[KZ] Hans G. Kaper and A. Zettl (eds.), Spectral theory of Sturm-Liouville operators, Proc. MayJune 1984 Workshop, ANL-84-73, Argonne National Laboratory, Argonne, IL, 1984.

[KKZ] Hans G. Kaper, M. K. Kwong, and A. Zettl, Singular Sturm-Liouville problems with nonnegative and indefinite weights, Monatsh. Math. 97 (1984), 177-189.

[L1] M. L. Lapidus, Valeurs propres du Laplacien avec un poids qui change de signe, C. R. Acad. Sci. Paris Sér. I. Math. 298 (1984), 265-268.

[L2] _ Fractal drum, inverse spectral problems for elliptic operators and a partial resolution of the Weyl-Berry conjecture, Trans. Amer. Math. Soc. 325 (1991), 465-529.

[L3] - Can one hear the shape of a fractal drum? Partial resolution of the Weyl-Berry conjecture, Geometric Analysis and Computer Graphics (P. Concus et al., eds.), Proc. Workshop held at the MSRI (Berkeley, May 1988), Math. Sci. Res. Inst. Publ., vol. 17, Springer-Verlag, New York, 1991, pp. 119-126.

[L4] Spectral and fractal geometry: from the Weyl-Berry conjecture to the Riemann zetafunction, Differential Equations and Mathematical Physics (C. Bennewitz, ed.), Proc. UAB Internat Conf. (Birmingham, March 1990), Academic Press, New York, 1992, pp. 151-182.

[LF1] M. L. Lapidus and J. Fleckinger-Pellé, Tambour fractal: vers une résolution de la conjecture de Weyl-Berry pour les valeurs propres du laplacien, C. R. Acad. Sci. Paris Sér. I Math. 306 (1988), 171-175.

[LF2] M. L. Lapidus and J. Fleckinger, The vibrations of a fractal drum, Differential Equations (Dafermos et al., eds.), Proc. "Equadiff 87", Lecture Notes in Pure Appl. Math., vol. 118, Dekker, New York, 1989, pp. 423-436.

[LP1] M. L. Lapidus and C. Pomerance, Fonction zêta de Riemann et conjecture de Weyl-Berry pour les tambours fractals, C. R. Acad. Sci. Paris Sér. I Math. 310 (1990), 343-348.

[LP2] - The Riemann zeta-function and the one-dimensional Weyl-Berry conjecture for fractal drums, Proc. London Math. Soc. (3) 66 (1993), 41-69.

[LoSt] L. H. Loomis and S. Sternberg, Advanced calculus, Addison-Wesley, Reading, MA, 1968. 
[Mt1] G. Métivier, Etude asymptotique des valeurs propres et de la fonction spectrale de problèmes aux limites, Thèse d'Etat, Nice, France, 1976.

[Mt2] _ Valeurs propres de problèmes aux limites elliptiques irréguliers, Bull. Soc. Math. France Mém. 51-52 (1977), 125-219.

[PI] Ȧ. Pleijel, Sur la distribution des valeurs propres de problèmes régis par l'équation $\Delta u+\lambda k(x, y) u=0$, Ark. Mat. Astr. och Fysick 29B (1942), 1-8.

[RS] M. Reed and B. Simon, Methods of modern mathematical physics, Vol. IV, Analysis of Operators, Academic Press, New York, 1978.

[We] H. F. Weinberger, Variational methods for eigenvalue approximation, CBMS Regional Conf. Ser. Appl. Math., vol. 15, SIAM, Philadelphia, PA, 1974.

Universite Toulouse 1, Place Anatole France, 31042 Toulouse Cedex, France

Department of Mathematics, The University of California, Sproul hall, Riverside, CALIFORNIA 92521-0135

E-mail address: lapidusQucr.math.edu 